\title{
Old active ingredients in new medicinal products: is the regulatory path coherent with patients' expectations?
}

Paola Minghetti ${ }^{1}$, Umberto M. Musazzi ${ }^{1}$, Antonella Casiraghi ${ }^{1}$, Paolo Rocco $^{1}$

${ }^{1}$ Department of Pharmaceutical Sciences, Università degli Studi di Milano, via G. Colombo, 71 20133 Milan, Italy.

\section{Corresponding Author:}

Prof. Paola Minghetti, PhD

Department of Pharmaceutical Science

Università degli Studi di Milano

Via Giuseppe Colombo, 71

20133 Milano (I)

Phone: +3902503 24639

Fax: +390250324657

Email: paola.minghetti@unimi.it

Short title: Old drugs in new medicinal products: regulatory paths

Keywords: Old drugs, Marketing Authorization, Hybrid applications, Drug repositioning, NonBiological Complex Drugs, fixed combinations

Teaser: $\quad$ The knowledge of the different regulatory pathways for marketing authorization can be useful to set up the most appropriate strategies to rationalize the medicament development and to assure the economic sustainability for payers.

\section{Conflict of Interest Statement:}

Authors have not any conflict of interest to declare 


\begin{abstract}
The rising costs of new medicinal products is a challenge to the economic sustainability of national healthcare systems in ensuring patients' access to therapies. EU and US legislators have provided regulatory pathways aimed at simplifying marketing authorisation applications of new medicinal products, in cases when safety and efficacy profiles can be derived from the data of alreadymarketed products. This article reviewed the different regulatory pathways towards the marketing authorisation of new medicinal products containing old drug substances and intended to improve the therapeutic value of a treatment, to obtain a new therapeutic indication (drug repositioning) or to ensure the same therapeutic value of a reference product at lower costs.
\end{abstract}




\section{Introduction}

The regulation of the development, production and marketing of new medicinal products is founded on the assurance of citizens' health, stating the requirements that manufacturers and Marketing Authorization (MA) Holders must follow to guarantee product quality, safety and efficacy. In general, a technical dossier, based on a standardised format (Common Technical Document, CTD) [1] - detailing how the data provided by the Applicant meets those requirements - has to be submitted to the competent authority granting the MA, which is obtained if the product benefit-risk balance is evaluated to be positive.

The development of a medicinal product containing an active pharmaceutical ingredient (API) never used in humans (first-in-human) is an expensive and risky activity for pharmaceutical companies. Indeed, the costs incurred by pharmaceutical companies for placing a new medicinal product on the market have been estimated at 2.8 billion dollars, with a significant increase in the last decade [2]. Most of the expenditure is ascribable to the translation from pre-clinical to clinical studies needed to demonstrate efficacy and safety of a medicinal product according to the current regulatory framework. To acknowledge the MA Holder (MAH) for financial resources invested in the preauthorisation process, all medicinal products are covered by intellectual property rights, which, in the European Union (EU), are extended by the supplementary protection certificate (SPC). When patent protection is not possible or not enough, other mechanisms, such as exclusivity in the United States (US) or data exclusivity/marketing protection in the EU may be granted $[3,4]$.

Once intellectual property rights and other protection mechanism have expired, the development of therapeutic equivalent copies by the competition becomes a feasible strategy to reduce treatments' costs and improve the economic sustainability of healthcare systems. However, the fate of an "old" API may not be limited to that. Indeed, thanks to technological and biomedical progress, old drug substances may be used in new or innovative applications. They can be reformulated into a new product for (i) same therapeutic indications, to improve its therapeutic value or (ii) totally new therapeutic indications. As an example, from 1998 to 2004, 33\% of all biotechnological medicinal products authorized by the European Medicines Agency (EMA) showed significant technological innovativeness compared to already-marketed therapeutic alternatives [5]. The percentage decreases to $12 \%$ for non-biological medicinal products. Moreover, since 2014 a mean of 150 already-authorized medicinal products per year have received a favourable opinion for an 
orphan designation by the EMA, whereas less than 20 new orphan products per year have been authorized for a new marketing authorization [6].

The development of a new drug product starting from an old drug substance brings significant advantages from a regulatory point of view. In most of the cases, the information about efficacy and safety profiles of the drug substance is already available in the literature or in possession of Regulatory Authorities. Consequently, the extent of data that the Applicant has to provide for the assessment is reduced, and drug products can be authorized following an abridged application. The nature and extent of such data may vary, based on the type of the API (biological or non-biological), the intrinsic complexity of drug product and its therapeutic indications.

This article aims to review the different regulatory pathways applicable to obtain a MA in the EU for new medicinal products containing an old drug substance, summarising, at the same time, the similarities and differences with the US regulatory framework; specific attention has been paid to the regulation of therapeutically equivalent copies. Different scenarios that may arise (Figure 1), and for which the data required vary in consideration of the type of API and formulation, are considered and discussed:

1) development of novel medicinal products containing an old API, intended to be used for the same therapeutic indications of previously authorized therapies but reformulated to improve the therapeutic benefit/risk profile (Section 2);

2) development of novel medicinal products containing an old API reformulated to be used for a different therapeutic indication (drug repositioning) (Section 3);

3) development of copies with no therapeutic advantages compared to already authorized medicines but providing economic advantages (Section 4).

In most of the cases, the criticisms and the regulatory requirements related to such scenarios are relevant both for the MA of a novel medicinal product and when an extension of therapeutic indications is submitted as major post-authorisation variations of an existing dossier. The current manuscript is focused on the regulatory pathways applicable for MA of novel medicinal products. Critical discussions on extended variations are available otherwhere in the literature $[7,8]$. 
No consensus has been found so far on whether of the expressions "drug repositioning" and "drug repurposing" are to be considered as synonyms or attributed different nuances of meaning. Moreover, the term "drug" has been used both with the meaning of "drug substance" and "drug product" [9]. For clarity, in this discussion, the expression "drug repositioning" is used throughout with reference to a drug substance contained in an already authorized medicinal product (which may have been withdrawn from the market) reformulated to be used for a therapeutic indication different from the ones of the authorized medicinal product.

\section{Improvement of the therapeutic value}

Old drug products can be re-formulated to improve or to optimize their therapeutic efficacy with the same/similar therapeutic indications. For example, the same API can be formulated in a new pharmaceutical form or a new route of administration, which are innovative from the technological or biopharmaceutical point of view bringing an improvement of the clinical/therapeutic outcomes, or included in a fixed combination. In this context, the improvement of the therapeutic value can be related to superiority in both efficacy and safety of the drug product in comparison to existing therapies, as well add in the increase in patient adherence to a therapy.

\subsection{From conventional to innovative delivery systems}

In some cases, therapeutic improvement is attributable to a change in the delivery system and dosage form of the medicinal product. Long acting injectables of leuprolide resulted in an improvement of clinical efficacy for advanced prostatic cancer and endometriosis in comparison to conventional injections [10]. Inhaled liposomal amikacin has been authorized as an orphan drug in the EU since 2014 for the treatment of nontuberculous mycobacterial lung disease [11] and, since 2018, for the treatment of mycobacterium avium complex (MAC) lung disease in the US [12]. Compared to conventional i.v. amikacin injection, the inhaled liposomal formulation brought an improvement of the API's lung distribution and its penetration into biofilms and macrophages, increasing the antimicrobial efficacy of the treatment against mycobacteria and reducing adverse events [13]. In all those cases, the information provided in support of a line-extension or an abridged application was reduced compared to first-in-human products. The quality part related to the API may be reduced, whereas the quality part of CTD related to the drug product should be full, including 
all the information regarding physicochemical and technological characterization of the product and its critical quality attributes based on the intended use and route of administration [14]. Data included in the preclinical and clinical parts of the dossier are reduced, but should be sufficient to allow an evaluation, by Regulatory Authorities, of the efficacy and safety profiles of the product, based on its features, complexity of the dosage form and nature of therapeutic improvement. The corresponding regulatory pathways in the EU and in the US are the hybrid application under Article 10(3) of Directive 2001/83/EC [15] and the 505(b)(2) NDA application [16], respectively.

\subsection{Change of parenteral administration route}

A similar approach can be applied for supporting a change of parenteral route. As an example, in the EU, a hybrid application was used for the MA of some monoclonal antibodies, the formulation of which was re-designed to allow i.m./s.c. administration.

Trastuzumab, a well-known anticancer monoclonal antibody, was a first case. According to Article 19 and Annex I of Reg. (EC) No 1234/2008, a line-extension of a drug product for i.v. infusion (Herceptin ${ }^{\circledR}$ ) was authorized in 2013, introducing on the market the subcutaneous drug injection [17]. The improvement of therapeutic value was based on patients' self-administration yielding a higher compliance than the i.v. product [18]. Indeed, the possibility to self-administer the needed doses have been historically well accepted by the patients [19]. However, it is also noteworthy that the use of a s.c. formulation may not be a real-life improvement per se, since other factors may concur to the success of therapy (e.g., therapy costs, role of healthcare professionals) [20, 21]. Nevertheless, in the case of trastuzumab, the composition of the i.v. injection was re-formulated to meet the target product profile of the new administration route: the composition of the s.c. injection, the strength, and the regimen were modified. A recombinant human hyaluronidase was also included in the product composition to facilitate subcutaneous delivery of the high-volume ( 5 $\mathrm{mL}$ ) formulation. For the nature of this variation, the dossier submitted for the application presented similarities to those generally submitted for an abridged application. Specially, a full quality assessment was provided by the Applicant to demonstrate comparability and stability of the new formulation. Non-clinical studies aimed to investigate the impact of recombinant human hyaluronidase on drug dispersion and absorption through subcutis, enzyme biodistribution and toxicological pattern. Since pharmacology and toxicology of trastuzumab are well-characterized after i.v. injection, only local tolerance studies were conducted. For the clinical part, only two clinical 
studies were reported: a phase I study for dose-finding on healthy man volunteers and a noninferiority phase III study on patients. For the latter, it is interesting to note that only non-clinical and phase-I clinical results were available at the moment of the variation authorization in 2013 [22], while results of phase III studies were published in $2019[23,24]$.

\subsection{Different pharmaceutical forms for different administration routes}

A hybrid application can also be used for a medicinal product which is formulated in a different pharmaceutical form, intended for a different administration route. It is the case of transdermal patches to delivery oxybutynin, an antimuscarinic drug used in treatment of overactive bladder, that brought a reduction of anticholinergic adverse events (e.g., dry mouth) induced by $\mathrm{N}$ desethyloxybutynin, a drug metabolite produced by the first-pass metabolism of oral drug therapies [25].

It is also the case of buprenorphine-loaded implants approved for the substitution treatment for opioid dependence in clinically stable adult patients. In comparison to already-authorised drugloaded sublingual tablets, the long-acting implants assure a drug release for six months, allowing for a simplification of the therapeutic regimen, better therapy control, which is independent of patient's will and lower risk of abuse or misuse [26]. In the last decades, different long-acting implants containing buprenorphine were authorized by the FDA (e.g., Probuphine ${ }^{\circledR}$, Sublocade ${ }^{\circledR}$ ), while in the EU, the first implant containing buprenorphine (Sixmo ${ }^{\circledR}$ ) was authorized by the EMA, through a centralized procedure, only in 2019 [27]. Such drug products are rod implants made of biodegradable polymers [i.e., Probuphine ${ }^{\circledR}$, Sixmo $^{\circledR}$ : ethylene-vinyl acetate copolymer (EVA); Sublocade ${ }^{\circledR}$ : 50:50 poly(DL-Lactide-co-glycolide) polymer] in which the drug is dispersed. The implant is considered as a complex parenteral formulation due to the higher complexity of manufacturing process, and the higher impact of its critical quality attributes on product efficacy and safety in comparison to conventional dosage forms $[10,28]$. For example, failures in quality controls during manufacturing process may produce in vivo a breaking of the rod with the consequent dose dumping, with an increased risk for the safety of patients. For an example, in the case of $\mathrm{Sixmo}^{\circledR}$, the EMA required a full quality part in the CTD, including the physicochemical characterization of both drug and excipients, implant stability and sufficient durability to assure both implantation and removal, and data supporting the continuous steady-state release profile of the drug for six-month treatment period. Validation of the manufacturing process and its key-steps 
were described in detailed. As in case of the trastuzumab s.c. injection, the profile of the API itself is well-characterised and, therefore, no further pharmacodynamic studies were required. Nonclinical data were focused on assessing the drug pharmacokinetics and toxicological pattern, including long-term toxicity, local tolerance studies and risk of dose dumping. Moreover, toxicological profiles of EVA were also a matter of investigation. Finally, the clinical part of the dossier included seven clinical trials. Among them, three phase-Ill studies, in which a total of 309 patients were treated with Sixmo ${ }^{\circledR}$ for up to 6 months ( 1 implant cycle), were performed to evaluate safety and efficacy of the drug-loaded implant [27]. Placebo implants and oral, sublingual tablets were used as references.

\subsection{Novel fixed combination}

Another case when an abridged application can be used is novel fixed combinations (fixed dose combinations in the US). A fixed combination is a medicinal product containing at least two APIs combined to improve therapeutic efficacy or safety profiles in comparison to monotherapies [29]. These products are regulated, in the EU, by the Article 10b of Directive 2001/83/EC, and, in the US, by the Section 300.50 of the Title 21 of Code of Federal Regulations.

APIs included in the fixed combination can act on either the same pharmacological target or different targets with a synergic effect (e.g., an angiotensin-converting enzyme inhibitor and a diuretic). According to intended use, a novel fixed combination can be developed and tested as 1) a substitution therapy in controlled patients, to simplify daily multidrug regimen or 2) an add-on treatment for patients for which no efficacy treatments are available on the market.

In both cases, if the APIs are already present in authorized medicinal products, the non-clinical and clinical studies performed by the Applicant could be limited to the pharmacological and clinical performance of the fixed combination, since the efficacy and safety profiles of the individual APIs are already known. In this field, evidence base of fixed combination can be supported by clinical studies or published literature (including clinical guidelines), or a combination of both [29].In the first case, requirements are limited to the demonstration of bioequivalence in comparison to the existing medicines, containing the single API, used in combination. As an example, fixed combinations for treating hypertension (e.g., lisinopril + hydrochlorothiazide; perindopril + amlodipine + indapamide). 
In the second case, the Applicant is required to submit relevant data and clinical studies to demonstrate the improvement of therapeutic value and the benefit/risk ratio of a fixed combination versus monotherapies. For example, the authorized fixed combinations with up to three APIs for treating HIV (Atripla ${ }^{\circledR}$ : efavirenz + emtricitabine + tenofovir), and $V_{y x e o s^{\circledR}}$, a fixed combination of daunorubicin hydrochloride and cytarabine in a liposomal system.

Vyxeos ${ }^{\circledR}$ was authorized by the FDA in 2017 [30] and by the EMA in 2018 as an orphan medicine for the treatment of therapy-related acute myeloid leukaemia with myelodysplasia-related changes [31]. Unlike previous examples, Vyxeos ${ }^{\circledR}$ resulted in significant therapeutic advantages concerning existing liposomal formulations. Indeed, the rationale of this drug product is the synergic pharmacological effect of the two antineoplastic drugs when they are combined in optimal molar ratio 5:1 cytarabine: daunorubicin. Liposomal systems were designed to assure the loading of drug over time, assuring a prolonged drug circulation and maintenance of the drug ratio fixed in vivo. As other complex formulation, small changes in critical quality attribute may have a substantial impact on the performance of liposomes in vivo. Therefore, a full characterization of such lipid vesicles was included in the quality part of CTD in agreement with the EMA "Reflection paper on the data requirement for intravenous liposomal products developed concerning an innovator liposomal product" [32]. For instance, the selection of liposomal components was in-depth discussed in light of previous knowledge of the Applicant, and liposomal systems were fully characterized in terms of morphology, lamellarity, bi-lamellar structure, surface charge, trapped volume, phase transition temperature. In agreement with the EMA Guideline on fixed combinations [29], non-clinical studies were focused on assessing, in different animal models, efficacy and safety profile of liposomal fixed combination versus the free drug cocktail. The overall results also suggested that the liposomal systems modify the pharmacokinetics of the two APIs in comparison to the combined use of the reference products. Clinical data confirmed the superiority of the liposomal systems in terms of both pharmacokinetics and efficacy profile. Only two phase-II studies and a single phase-III study on 309 patients have been submitted as part of the dossier to assess benefit-risk balance of drug product. Overall clinical results confirmed the superiority of the proposed drug product both in terms of efficacy and safety compared to the standard 7+3 regimen of cytarabine and daunorubicin [33]. Data demonstrated that the combination of both drugs in a single nanocarrier permitted to reduce total drug dose in comparison to the reference, which was considered a positive clinical benefit. 


\section{Drug repositioning}

Even if no longer on the market, an old API can be repositioned to be re-authorized for new therapeutic indications. In this case, innovation may not be found in the formulation, but in identification of a novel therapeutic target for which the benefit/risk balance of the old drug was considered positive [34]. On the one hand, drug repositioning can be due to a serendipitous discovery during preclinical, clinical and post-marketing studies of a medicinal product authorized for a different therapeutic indication. For example, topical and oral propranolol, a well-known betablocker, has been widely used for treating infantile haemangiomas since its effectiveness was serendipitously documented in 2008 [35, 36]. Sildenafil was initially studied as a drug for treating hypertension and angina pectoris. However, starting from observed common adverse effects, in the EU, it received a MA in 1998 as the first pharmacological therapy for erectile dysfunctions, becoming a blockbuster in a few years [37]. In 2005, sildenafil (Revatio ${ }^{\circledR}$ ) was then authorized by the EMA to treat adults and paediatric population from 1 year of age with pulmonary arterial hypertension [38]. Film-coated tablets and solutions for injection were developed.

Additionally, drug repositioning can be the results of an ad hoc pre-clinical and clinical research to find therapeutic solutions for rare or untreated diseases starting from molecules that have been withdrawn from the market due to safety concerns, as in the case of thalidomide. Withdrawn from the market in the 1960s because of its severe teratogenic adverse effect, it was re-authorized by the EMA in 2008 as first-line treatment of patients ( $\geq 65$ years) with untreated multiple myeloma in combination with melphalan and prednisone [39].

Since drug repositioning cannot be considered as a simple re-use of an old drug, the amount of data the applicant must provide may be significantly higher than previous cases. Indeed, non-clinical and clinical data cannot be completely derived from data on existing products or from the firstauthorization dossier. This is because safety and efficacy profiles of a repositioned drug may vary significantly for the pharmacological mechanism underlying the novel therapeutic indications. However, a hybrid application, or the 505(b)(2) NDA in the US, can still be used as regulatory pathway. Of course, the amount of abridged data varies case-by-case according to the type of drug repositioning proposed by the applicant. For example, relatively few clinical studies were submitted in addition to the extensive literature data to support the demonstration of the clinical efficacy of the propranolol, when proposed repositioning for treating infantile haemangiomas (Hemangiol ${ }^{\circledR}$ ) 
[40]. On the contrary, a high number of non-clinical and clinical data have been required for assessing the benefit/risk balance of thalidomide repositioning [39].

In comparison to other products authorized following a hybrid application, a more detailed research and development process to support the therapeutic application, including a suitable non-clinical and clinical dataset, is expected. Non-clinical and clinical studies should also be provided because of the pharmacodynamic rationale at the basis of the new use differs from previous knowledge. Moreover, repositioning approach is particularly interesting for developing a novel treatment for a rare disease. Indeed, in addition to reduced data required for the MA, development of a novel orphan drug product/indication was rewarded by the Regulatory Authorities with an extension of the market protection of the old medicinal product (for orphan indication) or the establishment of a market exclusivity period of 10 years for the authorization [41]. These provisions are particularly relevant for old APIs, for which patent protection is expired.

\section{Therapeutically equivalent copies}

After intellectual property rights and other protection mechanism have expired, the development of therapeutically equivalent copies may represent a desirable strategy to improve patients' access to pharmacological treatments and to reduce costs for healthcare systems. In this context, development of a copy should be focused on designing a product therapeutically equivalent to an already marketed medicinal product (the originator). The efficacy and safety profile can be derived from the originator one if the Applicant can provide demonstration of equivalence. However, according to the type of API or formulation, such an assessment may not be so easily performed, and data needed by the regulatory agency to determine the benefit/risk balance may increase proportionally to the complexity of the system (Figure 2 ).

\subsection{Small molecules}

If the copy contains already-authorized small molecules and has the same pharmaceutical form, strength and therapeutic indications of an already authorized medicinal product, the MA can be based on demonstration of bioequivalence as a surrogate of therapeutic equivalence. Consequently, a simplified dossier can be submitted to the regulatory agency. Such a regulatory pathway is adequately consolidated and harmonized worldwide. Considering that small APIs can generally be well-characterized by available analytical methods, the evaluation of benefit/risk balance of a copy 
of a marketed medicinal product is based on a two-pillar assessment. First, both the test and reference product should be pharmaceutical equivalents: they are identical in terms of API(s), dosage form, strength, route of administration. Then, they should be bioequivalent in vivo, i.e. no significant difference in their plasmatic bioavailability profile should be observed, from a statistic point of view, in human volunteers [42].

Procedures to be followed in the US (Abbreviated New Drug Application, ANDA) and the EU (generic application) are quite similar $[43,44]$. The extent of nonclinical and clinical data to be included in the CTD is reduced to demonstration of the bioequivalence in vivo. For quality assessment, the copy should be fully characterized, including proper in vitro studies to compare technological properties of the products. The impact of excipients, mainly those critical for their functionality-related characteristics, is also evaluated according to features of the drug product. In general, a small difference in qualitative/quantitative composition of excipients is accepted if no impact on drug bioavailability is expected and demonstrated by proper bioequivalence studies.

In some cases, bioequivalence may not be enough to assess therapeutic equivalence between test and reference products. In this context, the positions of the Regulatory Authorities are not harmonized. For example, the FDA states that ANDA applies to product copies intended for parenteral, ophthalmic, otic and topical use, only if the Q1/Q2 requirement - namely the qualitative (Q1) and quantitative (Q2) composition of test and reference products should be the same - is fulfilled $[45,46]$. Small differences in specific types of excipients (e.g., preservative, buffer, antioxidant, substance to adjust tonicity) are accepted if information demonstrating that differences do not affect the safety or efficacy of the proposed drug product are provided. On the other side, the position of the EMA is less restrictive because of a statement like Q1/Q2 requirement is not present in the European regulatory framework. Indeed, generic regulatory pathway can be followed for product copies intended for a parenteral use regardless of excipient composition. However, a biowaiver can be accepted by the EMA only if excipients do not interact with the API(s) or influence its biodistribution. In other cases, a bioequivalence study should be performed at least [47].

When a copy is not pharmaceutically equivalent to an authorized medicinal product or bioequivalence cannot be demonstrated, an abridged application can be used. This approach is used when bioequivalence cannot be considered as a surrogate of therapeutic equivalence. This aspect 
is particularly relevant for topically applied medicinal products for which systemic absorption is too low or negligible to perform a bioequivalence study [48].

To address this case, Regulatory Authorities adopted different strategies to support the Applicant in comparing the performance of a topically applied drug product versus an already marketed one. The FDA released different draft guidelines on specific drug products, e.g. semisolid preparation containing lidocaine [49] or patch containing diclofenac or lidocaine [50,51]. The EMA started a revision of the guideline on the quality and equivalence of topical products [52], and its draft exhibiting several similarities with FDA's position. Indeed, both Regulatory Authorities adopted a flexible approach based on the intrinsic complexity of drug product. For simple pharmaceutical dosage forms (e.g., solutions, ointments) with the same qualitative/quantitative composition of $\mathrm{API}(\mathrm{s})$ and excipients, risk of inequivalence is lower than for complex dosage forms (e.g., biphasic semisolid preparations) or for products with differences in excipients' composition. In the former case, bioequivalence can be assessed based on the equivalence of physicochemical properties and in vitro testing of relevant biopharmaceutical properties (e.g., drug release). This is the approach accepted by the FDA for lidocaine ointments [49]. The EMA introduced extended pharmaceutical equivalence, where therapeutic equivalence can be extrapolated from the equivalence of a quality pattern of test and reference products [52], a comparison based on in vitro release studies being also accepted. In the other cases, the equivalence of the two products should be assessed using proper biorelevant tests, such as permeation kinetics (in vitro permeation, stratum corneum sampling, pharmacokinetics bioequivalence) [53] or pharmacodynamic studies (vasoconstriction assay for corticosteroids, antiseptic and anti-infective studies). In this context, the development of surrogate in vitro, ex vivo or in vivo tests is desirable to speed up the authorization process and reduce costs. At the moment, only pharmacodynamic tests for corticosteroids and some antiinfective drugs are addressed by specific guidelines. Tests based on corticosteroid-induced vasoconstriction have been accepted by the FDA since 1995 to assess the equivalence of topically applied products containing such drug substances [54]. Otherwise, tests to compare in vitro permeation through skin have been accepted by the EMA as a surrogate of in vivo absorption [55]. In particular, in vitro permeation using Franz's diffusion cells were included in recent guidelines on (trans-)dermal dosage forms as tests to be performed during pharmaceutical development or as a surrogate of in vivo clinical studies $[52,56]$. If no surrogate tests can be used to assess therapeutic equivalence of two products, bioequivalence studies have to be performed at least to estimate the 
comparability of efficacy or safety profiles of test/reference products. The protocol design has to be adapted to allow a statistically significant comparison of plasmatic bioavailability. It is the case of the FDA guideline on lidocaine patches [50]. In addition to adhesion and a skin irritation comparative studies, a bioequivalence study with a dose three-time higher than the therapeutic one (three patches applied onto the skin) has to be performed.

\subsection{Biological medicinal products}

The development of therapeutic equivalent copies is much more challenging in the case of biological medicinal products [57]. Indeed, biological APIs show an intrinsic heterogenicity due to their manufacturing process and small changes in this process can have a considerable impact on the benefit/risk balance of the drug product. The same is true in the case of a "transfer" from one manufacturer to another, which, in general does not have access to full documentation on the original process.

The development of biological copies cannot be based only on the assessment of pharmaceutical equivalence and the bioequivalence as in the case of generics. Moreover, the pharmaceutical equivalence can be challenging due to API heterogenicity and difficulties in adopting sensitive analytical methods, which make demonstration of identity of the APIs not feasible. On the other hand, a similar pharmacokinetic profile is not enough to assure therapeutic equivalence between test and reference products since variations of protein structure may affect the pharmacodynamics without altering its biodistribution.

Focusing only on biological medicinal products containing biotechnology-derived proteins as active substance, "similarity" of the copy, so-called biosimilar, to the originator needs to be established "in terms of quality characteristics, biological activity, safety and efficacy based on a comprehensive comparability exercise" [58]. This "biosimilar" comparability exercise is based on the same scientific principles applied for evaluation of the impact of changes in the manufacturing process of a biological medicinal product and it is comprised of activities designed to investigate whether a copy and the originator are comparable [59]. In this context, "comparable" means the conclusion that the copy and the originator have highly similar quality attributes and that the transfer had no adverse impact on safety or efficacy, including immunogenicity, of the drug product. The conclusion of comparability is based on an analysis of product quality attributes and, if needed, nonclinical or clinical studies, number and extent of which may change depending on product classes and is 
generally determined on a case-by-case basis. After positive assessment of comparability, therapeutic equivalence can be assumed.

In the EU, the regulatory framework had to be upgraded to include a specific regulatory pathway for biosimilars, provided in Art. 10(4) of Directive 2001/83/EC. The EMA can authorize a biosimilar, following a centralized procedure, with an abridged dossier. Although the amount of data in the dossier is reduced compared to the originator's, it is still significantly more complex than in the case of generics. As for generics, full CTD modules 1, 2 and 3 are provided, with the added requirement that module 3 contains data from the comparability exercise. Unlike generics, for biosimilars modules 4 and 5 should consist of results from nonclinical and clinical comparability studies, where appropriate. The EMA releases product-specific guidelines, detailing how to assess similarity to test/reference products based on nonclinical (pharmacodynamic and toxicological studies) and clinical data (pharmacokinetics, safety and efficacy studies). The FDA applies a similar approach, through the Biologicals Price Competition and Innovation (BPCI) Act of 2009, which created an abbreviated authorization pathway for biosimilars.

However, the US and EU regulatory frameworks show some differences. Indeed, in the EU all APIs produced through biological or biotechnological manufacturing process are classified as biologics, while in the US the classification of biologics pertains only to natural polymeric structure comprising more than 40 alpha-amino acids [60]. FDA distinguishes between proteins and "chemically synthesized polypeptides", which are polypeptide chain up to 100 amino acids made entirely by chemical synthesis. This classification has recently changed as an effect of the "Deemed to be a License" provision of the BPCI Act, entered into force on 23 March 2020. The provision extends the scope of the PHS Act, to include any protein (except chemically synthesized polypeptides), so that "an application for a biological product approved under section 505 of the Federal Food, Drug, and Cosmetic Act (FDCA) (21 U.S.C. 355) will be deemed to be a license for the biological product under section 351 of the Public Health Service Act (PHSA) (42 U.S.C. 262)" [61]. As a result, products containing proteins now approved with a New Drug Application (NDA) or an ANDA will fall under the BPCIA.

APIs which are not proteins or polypeptides, such as low molecular weight heparins (LMWHs), on the contrary, are not affected by the modifications introduced by the "Deemed to be a License" provision, and differences between the EU and US remain. Copies of LMWHs are classified as 
biosimilars in the EU, but as generics in the US [62]. However, unlike other generics, the FDA established five requirements to demonstrate the sameness between the API of test and reference products that must be fulfilled to authorize an LMWH as a generic drug. In particular, the Applicants have to demonstrate the equivalence 1 ) of heparin source material and the mode of depolymerization; 2) of physicochemical properties of the API(s) by specific analytical methods; 3 ) in disaccharide building blocks, fragment mapping and sequence of oligosaccharide species; 4) in biological and biochemical assay; 5) of in vivo pharmacodynamic profiles.

Besides differences in the product classification, the difficulties in characterization of biologicalderived API(s) posed issues in therapeutic switch among product expected to be similar. Due to the similarity of their benefit/risk ratio and, therefore, their expected therapeutic equivalence, biosimilars may be considered interchangeable and/or substitutable, but the situation is different among the US and different EU countries [63].

\subsection{Non-biological complex drugs}

Although no consensus definition is still available, non-biological drug products showing high intrinsic complexity are generally classified as Non-Biological Complex Drugs (NBCDs). NBCDs are non-biological medicinal products, the API of which is "not a homomolecular structure but consists of different (closely related and often nanoparticulate) structures that cannot be isolated and fully quantitated, characterized and/or described by physicochemical analytical means" [64]. The use of the term NBCD was initially limited to intravenous iron products, liposomal systems and glatiramoids $[65,66]$, but it was later extended to include other classes, based on the complexity of their non-biological API (e.g., polymeric compounds, complex mixtures of APIs), formulation (e.g., liposomes, colloids), dosage form (e.g., transdermals, modified release injectables), route of delivery or combination with a Medical Device (so-called drug-device combinations, e.g., auto injectors, metered dose inhalers) [28].

The interest of regulatory and scientific community for a proper assessment of benefit-risk balance of complex medicinal products, in particular with a view of marketing copies, has risen in the last decades pushed by technological progress (e.g., nanotechnology) and its biomedical applications (e.g., nanomedicine). Indeed, therapeutic equivalence of an NBCD copy cannot be evaluated with the same approach used for medicinal products containing small molecules. Like biosimilars, the pharmaceutical equivalence of NBCDs containing complex API cannot be fully assessed since the 
demonstration of API sameness (both qualitatively and quantitatively) in comparison to the originator, may not be feasible with the same procedure used in the case of small molecules. This is the case of glatiramer acetate, a heterogeneous mixture of not fully characterized synthetic polypeptides authorized both in the EU and US for the treatments of relapsing forms of multiple sclerosis [66].

In general, small changes in manufacturing process of a NBCD have the potential to significantly alter the mixture in terms of peptide ratio if the process is not kept under control. Analogously, biopharmaceutical properties of the product can be significantly altered by variations of their excipient composition, their functionality-related characteristics or their manufacturing process, as in case of intravenous iron product and PLGA-based microparticle depot formulations [67, 68]. In other cases, therapeutic equivalence cannot be assessed based on bioequivalence since the drug carrier itself significantly influences both the pharmacology and toxicology of the loaded API(s) (e.g., nanomedicine products). For example, changes in the excipients' composition of intravenous iron products have modified the physicochemical properties of drug-excipient complex (e.g., dextranbased complex are more stable than disaccharide-based one), and also drug release in vivo and toxicological pattern $[69,70,71]$.

Considering the peculiarities of NBCDs, and the lack of a specific regulation, the authorization of their first copies proved challenging for Regulatory Authorities, though this was addressed differently in the US end the EU. In both territories, an abridged application is usually required, which the Applicants need to integrate with additional equivalence data to better characterize the quality, safety and efficacy profile. Moreover, additional efforts have been made by the related Pharmacopoeias to provide monographs and protocols on the physicochemical characterization NBCDs and their functionality-related characteristics [72].

In the EU, where NBCDs are not necessarily assessed through a centralised procedure, determination of the benefit-risk balance is based on a case-by-case evaluation, and, in some cases, an approach symmetric to that of biosimilars has been applied [28]. The EMA has been releasing reflection papers on nanomedicine products, namely liposomal systems, iron-core nanoparticles, micellar systems (i.e., conventional and block copolymer micellar systems), and coated nanosystems, but not systematically reviewing all NBCDs [32]. In the available documents, the agency detailed all the studies that the Applicant should include in the quality, safety and efficacy 
parts of the CTD to accurately weigh its benefit/risk balance and to demonstrate the product similarity in comparison to the reference one. For the marketing authorisation of NBCDs copies, either generic or hybrid applications can be used, depending on the intrinsic complexity of the drug products. For instance, PLGA-based microparticle depot formulations have been authorized as generics, despite their intrinsic complexity, while glatiramer acetate copies followed a hybrid application.

In the USA, regulators and applicants can take advantage of a centralization of the authorization process. The FDA has been releasing Product-Specific Guidances (PSGs) for a growing number of complex products, to support development and approval of safe and effective complex generic drug products [73]. For complex-API NBCDs, guidelines provide the criteria to assess API sameness, in comparison with originator. In the case of glatiramer acetate, the PSG states that API sameness should be demonstrated based on the equivalence of fundamental reaction scheme, physicochemical properties and structural signatures for polymerization and depolymerization and specific biological assay. If all these requirements are fulfilled, the copies can be authorized following an ANDA procedure and, therefore, they are handled as other generic drugs [28].

Different authorization approaches also affect interchangeability of NBCD copies and their substitution at dispensing level without physician explicit prior consent. In the US, complex generic drug products included in the FDA Orange book (code " $A$ ") are interchangeable with each other and can be substituted as other generic products. In the EU, the EMA does not take a position on interchangeability during the authorisation process. These issues are left to local regulatory agencies, the decision of which is strongly related to the procedure followed to obtain the MA: in the case of a generic procedure, the interchangeability is generally acknowledged. If a hybrid procedure is followed, however, local regulators may take different decisions on interchangeability.

If scientific knowledge is not developed enough to allow a full characterization of the NBCD medicinal products, the fact that too lax a regulatory pathway may be applied can expose the patients to increased health risks. Indeed, in the past, copies of intravenous iron products have been authorized as generic products for years until severe adverse effects after clinical substitution were recorded [74], when technology had progressed to the point of allowing a clear understanding of the complexity of the product and its impact on efficacy and safety profiles. The overall results 
demonstrated that such products could not be interchangeable without the physician's control and surveillance.

Although the European regulatory framework has been extended in the last decades to tackle critical issues related to the increased complexity of novel medicinal products, interchangeability of the NBCD copies remains a challenging issue. On the one hand, comparability studies improved the expertise of Regulatory Authorities and the clinical community on real-world risks of new drug products in comparison to existing therapeutic-equivalent treatments. On the other hand, such studies are performed in comparison to one specific reference product and not versus all therapeutic-equivalent copies, so that what may be called "cross-comparability" is not assessed. Indeed, although different copies can be equivalent to the same reference product, they may not be equivalent to each other due to the small changes in quality, safety and efficacy profile. A similar situation was observed for copies of medicines with narrow therapeutic index, e.g. Levothyroxine generics $[75,76]$, or for some NBCD $[67,70]$. Therefore, unlike most small-molecule copies for which pharmaceutical equivalence can be assessed, therapeutic equivalence among different NBCD copies cannot be assumed based on the results obtained concerning a shared originator. In this context, new regulatory tools are needed, together with an improved clinical knowledge about the interchangeability of NBCD copies. Rather than the creation of a new regulatory pathway, efforts of the EU legislator should be focused on speeding up the harmonization of procedures and protocols to authorize NBCD copies, avoiding the case-by-case assessments for products expected to be pharmaceutically and therapeutically similar. The strategy applied by the FDA to glatiramer acetate and LMWHs represents a possible starting point. Establishment of product-specific sets of comparability studies, including analytical methodologies and protocols, can harmonize the information submitted to the Regulatory Authorities to assess quality, safety and efficacy of copies of the same NBCD originators. Therefore, resulting harmonized datasets can be shared between the EMA and HMA associates, allowing to provide meta-analysis and better estimate the interchangeability of the marketed copies.

\section{Conclusion}

The expectation of patients on medicines can be summarised into two main aspects. On the one hand, patients expect that the pharmacological treatments they assume are effective and safe. In this context, the regulatory frameworks currently in force both in the EU and the US aim at fulfilling 
this expectation by establishing different pathways and requirements based on the benefit-risk balance of products. On the other hand, they expect medicines to be available on the market and economically accessible.

Several factors may influence the availability and accessibility of medicinal products. On the pharmacoeconomic side, too high costs of development may result either in a negative return on investment (which can lead, for example, to a withdrawal from the market), or in too high a price (which would lead to an inaccessible product for most patients). On the regulatory side, overprotective legislation could potentially prevent medicinal products with a positive benefit-risk balance to reach the market. A regulation that is too lax, or producing an increase of off-label prescriptions, may negatively impact the benefit-risk balance, exposing citizens to avoidable health risks and is not an acceptable option to speed up the marketing of innovative drug products. On the other hand, an overly restrictive regulation may imply a huge amount of nonclinical and clinical data to be submitted by the Applicant, even for medicinal products containing well-known or wellcharacterized drug substances, where they could be unnecessarily based on the available information. This scenario leads to higher costs of development and an unsustainable situation for both pharmaceutical companies and national health systems, ultimately resulting in a higher risk of shortages and withdrawals [77].

Regulatory requirements should be based on scientific and technical progress, the impact of which on public health assurance and medicinal products' economic sustainability should be carefully assessed until a proper balance between health protection and medicine accessibility is reached.

Such a balance is particularly important for medicines containing "old" drug substances. Although they have been on the market for long, they can also produce therapeutic innovation. As discussed, development and marketing of new medicinal products containing old drug substances can be a valid strategy aimed at improving the therapeutic value of pharmacological therapies, finding new treatments for rare diseases, or reducing costs that national healthcare systems have to support for ensuring patients' access to therapies. In comparison to first-in-human medicinal products, the ones based on old drug substances benefit from lower development costs and faster regulatory pathways for MA, since part of preclinical and clinical data may already be available or can be extrapolated from pharmacovigilance data of other products. These aspects can be particularly important in the 
case of rare pathologies, where the economic sustainability of a medicinal product is strongly affected by the small number of patients.

The regulatory frameworks in place in the EU and the US appear to be flexible enough to foster innovation and to protect citizens' health. The establishment of a hybrid application in the EU and similar authorization procedures in the US has allowed for rationalization of scientific and economic investments for new medicinal products reducing the extent of non-clinical/clinical data needed but, at the same time, maintaining the same standards of first-in-human products in assessing the benefit-risk balance of treatments. Moreover, several economic and regulatory incentives have been established to support development of new medicinal products, as in the case of orphan drugs, others to improve economic sustainability for manufacturers, in the post-marketing phases (e.g., specific provisions on intellectual property protections, including additional years of data exclusivity/market protection) [78, 79].

However, critical issues are still present. For example, unavailability of pharmacological treatments for rare diseases or delays between the expiration of intellectual property protections and the marketing of complex copies suggest that regulatory frameworks still have to find an optimal balance between regulatory assessment of the benefit-risk balance and economic sustainability. In the case of rare diseases, nonclinical models have been implemented for predicting the therapeutic value of new or repositioned drug products [80]. In the case of complex copies, the use of in vitro comparability studies in place of clinical studies could be a good strategy for reducing pre-marketing economic investments. The assessment remains linked to case-by-case criteria that limit information sharing, the determination of the significance of the "intent to treat" for new medicines, and the comparison among products and copies expected to be therapeutic equivalent. Moreover, the lack of a well-established scientific knowledge of in vitro/in vivo correlation for complex drugs - together with the challenge of characterising them - affects the assessment of API sameness and the biorelevance of in vitro test, hindering development of lower-cost copies.

Finally, a stronger collaboration between academia and pharmaceutical industries is desirable through all phases of medicinal product development and post-marketing surveillance. It would improve the industrial applicability of research results and fill the existing gaps in the benefit/risk assessment of innovative complex products. 


\begin{tabular}{ll} 
Abbreviations \\
ANDA: & Abbreviated New Drug Application \\
API: & Active pharmaceutical ingredients \\
BPCl: & Biologicals Price Competition and Innovation \\
CTD: & Common Technical Document \\
EMA: & European Medicines Agency \\
EVA: & Ethylene-Vinyl Acetate copolymer \\
FDA: & Food and Drug Administration \\
HMA: & Heads of Medicines Agencies \\
LMWH: & Low Molecular Weight Heparins \\
MA: & Marketing Authorization \\
MAC: & Mycobacterium Avium Complex \\
MAH: & Marketing authorization holder \\
NBCD: & Non-Biological Complex Drug \\
NDA: & New Drug Application \\
PLGA: & Poly(Lactic-co-Glycolic Acid) \\
\hline
\end{tabular}




\section{References}

1. The International Council for Harmonization of Technical Requirements for Pharmaceuticals for Human Use (ICH). M4 Common Technical Document (CTD). Accessible at: https://www.ich.org/page/ctd. [web access: April $8^{\text {th }} 2020$ ].

2. Di Masi JA, Grabowski HG, Hansen RW. Innovation in the pharmaceutical industries: new estimates of R\&D costs. J. Health Econ. 2016; 47: 20-33.

3. Directive 2001/83/EC of the European Parliament and of the Council of 6 November 2001 on the Community code relating to medicinal products for human use, as amended by Dir. 2004/27/EC. Article 10.

4. U.S. Food and Drug Administration (FDA). Frequently Asked Questions on Patents and Exclusivity. Available at: https://www.fda.gov/drugs/development-approval-processdrugs/frequently-asked-questions-patents-and-exclusivity [web access: April 16th, 2020].

5. Motola D, De Ponti F, Poluzzi E, Martini N, Rossi P, Silvani MC, Vaccheri A, Montanaro N. An update on the first decade of the European centralized procedure: how many innovative drugs? Br J Clin Pharmacol 2006, 62: 610-616.

6. European Medicines Agency (EMA). Annual report 2018: The European Medicines Agency's contribution to science, medicines and health in 2018. May 2018.

7. Langedijk J, Whitehead CJ, Slijkerman DS, Leufkens HGM, Schutjens M-H DB, MantelTeeuwisse AK. Extensions of indication throughout the drug product lifecycle: a quantitative analysis. Drug Discovery Today 2016; 21: 348-355.

8. Murteira S, Millier A, Ghezaiel Z, Lamure M. Drug reformulations and repositioning in the pharmaceutical industry and their impact on market access: regulatory implications. Journal of Market Access \& Health Policy 2014; 2:1, 22813, DOI: 10.3402/jmahp.v2.22813.

9. Langedijk J, Mantel-Teeuwisse AK, Slijkerman DS, Schutjens MH. Drug repositioning and repurposing: terminology and definitions in literature. Drug Discov. Today 2015; 20 :10271034.

10. Selmin F, Musazzi UM, Magri G, Rocco P, Cilurzo F, Minghetti P. Regulatory aspects and quality controls of polymer-based parenteral long-acting drug products: the challenge of approving copies. Drug Discovery Today 2020; 25: 321-329 
11. European Medicines Agency (EMA). Public summary of opinion on orphan designation. Amikacin sulfate for the treatment of nontuberculous mycobacterial lung disease. Available at: https://www.ema.europa.eu/en/medicines/human/orphan-designations/eu3141259 [web access: January 29th, 2020].

12. U.S. Food and Drug Administration (FDA). Arikayce ${ }^{\circledR}$, labelling. Available at: https://www.accessdata.fda.gov/drugsatfda_docs/nda/2018/207356Orig1s000Lbl.pdf [web access: January 29th, 2020].

13. Zhang J, Leifer F, Rose S, Chun DY, Thaisz J, Herr T, Nashed M, Joseph J, Perkins WR, DiPetrillo K. Amikacin liposome inhalation suspension (ALIS) penetrates non-tuberculous mycobacterial biofilms and enhances amikacin uptake into macrophages. Front. Microbiol. 2018; 9: 915. https://doi.org/10.3389/fmicb.2018.00915.

14. European Medicines Agency (EMA). Guideline ICH Q8 (R2) on Pharmaceutical development. EMEA/CHMP/167068/2004. Jun 2017.

15. Directive 2001/83/EC of the European Parliament and of the Council of 6 November 2001on the Community code relating to medicinal products for human use, as amended. Article 10(3)

16. 21 C.F.R. $\S 314.54$.

17. European Medicines Agency (EMA). Herceptin ${ }^{\circledR}$. CHMP variation assessment report (EMA/CHMP/751770/2012/corr1). Available at: https://www.ema.europa.eu/en/documents/variation-report/herceptin-h-c-278-x-0060epar-assessment-report-extension_en.pdf [web access: July 30th, 2019].

18. Pivot X, Spano JP, Espie M, Cottu P, Jouannaud C, Pottier V, Moreau L, Extra JM, Lortholary A, Rivera P, Spaeth D, Attar-Rabia H, Benkanoun C, Dima-Martinez L, Esposito N, Gligorov J. Patients' preference of trastuzumab administration (subcutaneous versus intravenous) in HER2-positive metastatic breast cancer: Results of the randomised MetaspHer study. European Journal of Cancer 2017; 82: 230-236.

19. Jin J-F, Zhu L-L, Chen $M$, Xu H-M, Wang H-F, Feng X-Q, Zhu X-P, Zhou Q. The optimal choice of medication administration route regarding intravenous, intramuscular, and subcutaneous injection. Patient Preference and Adherence 2015; 9: 923-942. 
20. Richardson SJ, Brooks HL, Bramley G, Coleman JJ. Evaluating the Effectiveness of SelfAdministration of Medication (SAM) Schemes in the Hospital Setting: A Systematic Review of the Literature. PLoS ONE 2014; 9: e113912.

21. Lin J, Sklar GE, Oh VMS, Li SC. Factors affecting therapeutic compliance - A review from the patient's perspective. Ther. Clin. Risk Manag. 2008; 4: 269-286.

22. European Medicines Agency (EMA). Herceptin ${ }^{\circledR}$. Procedural steps taken and scientific information after the authorisation. Available at: https://www.ema.europa.eu/ en/documents/procedural-steps-after/herceptin-epar-procedural-steps-taken-scientificinformation-after-authorisation en.pdf [web access: April $\left.8^{\text {th }} 2020\right]$.

23. Ismael G, Hegg R, Muehlbauer S, Heinzmann D, Lum B, Kim S-B, Pienkowski T, Lichinitser M, Semiglazov V, Melichar B, Jackisch C. Subcutaneous versus intravenous administration of (neo)adjuvant trastuzumab in patients with HER2-positive, clinical stage I-III breast cancer (HannaH study): a phase 3, open-label, multicentre, randomised trial. The Lancet Oncology 2012; 13: 869-878.

24. Jackisch C, Stroyakovskiy D, Pivot X, Ahn JS, Melichar B, Chen SC, Meyenberg C, Al-Sakaff N, Heinzmann D, Hegg R. Subcutaneous vs intravenous trastuzumab for patients with ERBB2positive early breast cancer: final analysis of the HannaH phase 3 randomized clinical trial. JAMA Oncol. 2019; 5: e190339.

25. Appell RA, Chancellor MB, Zobrist RH, Thomas H, Sanders SW, Pharmacokinetics, metabolism, and saliva output during transdermal and extended-release oral oxybutynin administration in healthy subjects. Mayo Clin Proc. 2003; 78: 696-702.

26. Itzoe MK, Guarnieri M. New developments in managing opioid addiction: impact of a subdermal buprenorphine implant. Drug Des Devel Ther 2017; 11: 1429-1437.

27. European Medicines Agency (EMA). Sixmo ${ }^{\circledR}$ EPAR. Available at: https://www.ema.europa.eu/en/medicines/human/EPAR/sixmo $\left[\right.$ web access: April $8^{\text {th }}$ 2020].

28. Rocco P, Musazzi UM, Franzè S, Minghetti P. Copies of nonbiological complex drugs: generic, hybrid or biosimilar? Drug Discovery Today 2019; 24: 250-255.

29. European Medicines Agency (EMA). Guideline on clinical development of fixed combination medicinal products. EMA/CHMP/158268/2017. London, 2017. 
30. U.S. Food and Drug Administration (FDA). Vyxeos ${ }^{\circledR}$. Multi-discipline review. Available at: https://www.accessdata.fda.gov/drugsatfda_docs/nda/2017/2094010rig1s000Multidiscipl ineR.pdf [web access: April 15th, 2020].

31. European Medicines Agency (EMA). Vyxeos $^{\circledR}$. EPAR - Product information. Available at: https://www.ema.europa.eu/en/documents/assessment-report/vyxeos-epar-publicassessment-report_en.pdf [web access: July 31st, 2019].

32. Musazzi UM, Marini V, Casiraghi A, Minghetti P. Is the European regulatory framework sufficient to assure the safety of citizens using health products containing nanomaterials? Drug Discovery Today 2017; 22: 870-882.

33. Maakaron JE, Mims AS. Daunorubicin-cytarabine liposome (CPX-351) in the management of newly diagnosed secondary AML: a new twist on an old cocktail. Best Practive \& Research Clinical Haematology 2019; 32: 127-133.

34. Minghetti P, Lanati EP, Godfrey J, Solà-Morales O, Wong O, Selletti S. From Off-Label to Repurposed Drug in Non-Oncological Rare Diseases: Definition and State of the Art in Selected EU Countries. MA@PoC 2017; 1: e87-e97.

35. Solman L, Glover M, Beattie P, Buckley H, Clark S, Gach J, Giardini A, Helbling I, Hewitt R, Laguda B, Langan S, Martinez A, Murphy R, Proudfoot L, Ravenscroft J, Shahidullah H, Shaw L, Syed S, Wells L, Flohr C. Oral propranolol in the treatment of proliferating infantile haemangiomas: British Society for Paediatric Dermatology consensus guidelines. Br. J. Dermatol. 2018; 179: 582-589.

36. Casiraghi A, Musazzi UM, Rocco P, Franzè $S$, Minghetti P. Topical Treatment of Infantile Haemangiomas: A Comparative Study on the Selection of a Semi-Solid Vehicle. Skin Pharmacology and Physiology 2016; 29: 210-219.

37. Goldstein I, Burnett AL, Rosen RC, Park PW, Stecher VJ. The Serendipitous Story of Sildenafil: An Unexpected Oral Therapy for Erectile Dysfunction. Sex Med. Rev. 2019; 7: 115-128.

38. European Medicines Agency (EMA). Revatio ${ }^{\circledR}$ https://www.ema.europa.eu/ en/medicines/human/EPAR/revatio\#product-information-section [web access: July 7th, 2019].

39. European Medicines Agency (EMA). Thalidomide Celgene (previously Thalidomide Pharmion). EPAR - Product information. Available at: 
https://www.ema.europa.eu/en/medicines/human/EPAR/thalidomide-celgene-previouslythalidomide-pharmion [web access: July 7th, 2019].

40. European Medicines Agency (EMA). Hemangiol ${ }^{\circledR}$. EPAR - Product information. Available at: https://www.ema.europa.eu/en/medicines/human/EPAR/hemangiol [web access: April 16th, 2020].

41. Franco P. Orphan drugs: the regulatory environment. Drug Discovery Today 2013; 18: 163172.

42. Zhang $\mathrm{X}$, Zheng $\mathrm{N}$, Lionberger RA, Yu LX. Innovative approaches for demonstration of bioequivalence: the US FDA prospective. Ther Deliv 2013; 4: 725-740.

43. 21 C.F.R. $§ 314.92$.

44. Directive 2001/83/EC of the European Parliament and of the Council of 6 November 2001 on the Community code relating to medicinal products for human use, as amended. Article 10.

45. 21 C.F.R. §314.94(a)(9)(iii).

46. U.S. Food and Drug Administration (FDA). Guidance for Industry. Controlled correspondence related to generic drug development. Draft guidance. November 2017.

47. European Medicines Agency (EMA). Guideline on the investigation of bioequivalence (CPMP/EWP/QWP/1401/98 Rev. 1/ Corr **). London, 2010.

48. Chang R-K, Raw A, Lionberger R, Yu L. Generic development of topical dermatologic products: Formulation development, process development, and testing of topical dermatologic products. AAPS Journal 2013; 15: 41-52.

49. U.S. Food and Drug Administration (FDA). Draft guidance on Lidocaine (Ointment, topical). Rev. Dec. 2016.

50. U.S. Food and Drug Administration (FDA). Draft guidance on Lidocaine (patch, topical). Rev. Oct 2016.

51. U.S. Food and Drug Administration (FDA). Draft guidance on diclofenac epolamine (patch, topical). Rev. Mar 2012.

52. European Medicines Agency (EMA). Draft guideline on quality and equivalence of topical products (CHMP/QWP/708282/2018). London, 2018. 
53. Leal LB, Cordery SF, Delgado-Charro MB, Bunge AL, Guy RH. Bioequivalence methodologies for topical drug products: in vitro and ex vivo studies with a corticosteroid and anti-funginal drug. Pharm Res 2017; 34: 730-737.

54. U.S. Food and Drug Administration (FDA). Guidance for Industry. Guidance Topical Dermatologic Corticosteroids: in vivo bioequivalence. Jun 1995.

55. Cilurzo F, Musazzi UM, Franzè S, Fedele G, Minghetti P. Design of in vitro skin permeation studies according to the EMA guideline on quality of transdermal patches. Eur J Pharm Sci 2018; 125: 86-92.

56. European Medicines Agency (EMA). Guideline on Quality of Transdermal Patches. EMA/CHMP/QWP/608924/2014. London, 2014.

57. Minghetti P, Rocco P, Cilurzo F, Del Vecchio L, Locatelli F. The regulatory framework of biosimilars in the European Union. Drug Discovery Today 2012; 17: 63-70.

58. European Medicines Agency (EMA). Guideline on similar biological medicinal products (CHMP/437/04 Rev 1, 23 October 2014)

59. International Council for Harmonisation of Technical Requirements for Pharmaceuticals for Human Use (ICH). Comparability of Biotechnological/Biological Products subject to changes in their manufacturing process -Topic Q5E, Step 4 (18 November 2004).

60. U.S. Food and Drug Administration (FDA). Definition of the Term "Biological Product" (FDA2018-N-2732) (2018).

61. U.S. Food and Drug Administration (FDA). The "Deemed to be a License" Provision of the BPCl Act: Questions and Answers. Guidance for Industry (March 2020). https://www.fda.gov/media/135838/download. Last accessed on 15 April 2020.

62. Minghetti P, Cilurzo F, Franzè S, Musazzi UM, Itri M. Low Molecular weight heparins copies: are they considered to be generics or biosimilars? Drug Discovery Today 2013; 18: 305-311.

63. Minghetti $P$, Rocco $P$, Schellekens $H$. The constrained prescription, interchangeability and substitution of biosimilar. Nature Biotechnology 2015; 33: 688-689.

64. Crommelin DJ, de Vlieger JS, Weinstein V, Muhlebach S, Shah VP, Schellekens H. Different pharmaceutical products need similar terminology. AAPS Journal 2014; 16: 11-14. 
65. Borchard G, Fluhmann B, Muhlebach S. Nanoparticle iron medicinal products Requirements for approval of intended copies of non-biological complex drugs (NBCD) and the importance of clinical comparative studies. Regulatory Toxicology and Pharmacology 2012; 64: 324-328.

66. Rocco P, Eberini I, Musazzi UM, Franzè S, Minghetti P. Glatiramer acetate: a complex drug beyond biologics. European Journal Pharmaceutical Sciences 2019; 133: 8-14.

67. Di Francesco T, Borchard PG. Iron sucrose: assessing the similarity between the originator drug and its intended copies. Annals of the New York Academy of Sciences 2017; 1407: 6374.

68. Garner J, Skidmore S, Park H, Park K, Choi S, Wang Y, Beyond Q1/Q2: the impact of manufacturing conditions and test methods on drug release from PLGA-based microparticle depot formulations, J. Pharm. Sci. 2018; 107: 353-361.

69. Geisser P, Burckhardt S. The pharmacokinetics and pharmacodynamics of iron preparations. Pharmaceutics 2011; 3: 12-33.

70. Toblli EJ, Cao G, Oliveri L, Angerosa M. Differences between original intravenous iron sucrose and iron sucrose similar preparations. Drug Res. 2009; 59: 176-190.

71. Martin-Malo A, Borchard G, Fluhmann B, Mori C, Silverberg D, Jankowska EA. Differences between intravenous iron products: focus on treatment of iron deficiency in chronic heart failure patients. ESC Heart failure 2019; 6: 241-253.

72. Borchard G. Complexity in the making: non-biological complex drugs (NBCDs) and the pharmacopoeias. GaBI Journal 2016; 5: 36-41.

73. U.S. Food and Drug Administration (FDA). Upcoming Product-Specific Guidances for Complex Generic Drug Product Development. https://www.fda.gov/drugs/guidances-drugs/ upcoming-product-specific-guidances-complex-generic-drug-product-development [web access: April 16th, 2020].

74. European Medicines Agency (EMA). Intravenous iron-containing medicinal products. Article 31 referral (EMEA/H/A-31/1322). Available at: https://www.ema.europa.eu/en/medicines/ human/referrals/intravenous-iron-containing-medicinal-products [web access: July 30th, 2019]. 
75. Concordet D, Gandia P, Montastruc J-L, Bousquet-Mélou A, Lees P, Ferran A, Toutain P-L. Levothyrox ${ }^{\circledR}$ New and Old Formulations: Are they Switchable for Millions of Patients? Clinical Pharmacokinetics 2019; 58:827-833.

76. Hennessey JV. Generic vs Name Brand L-Thyroxine Products: Interchangeable or Still Not? J. Clin. Endocrinol. Metab. 2013; 98(2):511-514.

77. Musazzi UM, Di Giorgio D, Minghetti P. New regulatory strategies to manage medicines shortages in Europe. Int. J. Pharm. 2020; 579: 119171.

78. Verbaander C, Rooman I, Meheus L, Huys I. On-Label or Off-Label? Overcoming Regulatory and Financial Barriers to Bring Repurposed Medicines to Cancer Patients. Front. Pharmacol. 10:1664. doi: 10.3389/fphar.2019.01664.

79. Nayroles G, Frybourg S, Gabriel S, Kornfeld A, Antoñanzas-Villar F, Espín J, Jommi C, Martini N, de Pouvourville G, Tolley K, Wasem J., Toumik M. Unlocking the potential of established products: toward new incentives rewarding innovation in Europe. Journal of market access \& health policy $2017 ; 5,1298190$.

80. Sheean ME, Stoyanova-Beninska V, Capovilla G, Duarte D, Hofer MP, Hoffmann M, Magrelli A, Mariz S, Tsigkos S, Shaili E, Polsinelli B, Ricciardi M, Bonelli M, Balabanov P, Larsson K, Sepodes B. Nonclinical data supporting orphan medicinal product designations: lessons from rare neurological conditions. Drug Discov Today 2018; 23:26-48. 


\section{Figure captions}

Figure 1 - Possible scenarios for the development of new medicinal products containing old active pharmaceutical ingredients.

Figure 2 - European regulatory pathways that can be followed for the MA of a new drug product that is a copy of an already marketed one. 